\title{
Conocimiento Didactico del Contenido Curricular, el diseño en la formación permante de profesores de Químca
}

\author{
Didactic knowledge of the content, the design in the permanent formation of \\ teacher's of ckemistry
}

Juan G. Perilla J. ${ }^{1}$ y Diana Lineth Parga Lozano ${ }^{2}$

\begin{abstract}
${ }^{1}$ Estudiante del programa de Maestria en Docencia de la Química. Universidad Pedagógica Nacional. ${ }^{2}$ Docente del Departamento de Química. Universidad Pedagógica Nacional; Directora del proyecto. jgperilla@gmail.com, dparga@pedagogica.edu.co
\end{abstract}

\section{Resumen}

La formación permanente de docentes, es en la actualidad, objetivo reconocido e induso prioritario de muchas administraciones educativas así como de numerosas instituciones y organismos, oficiales y privados, sensibles a esta necesidad. Se trata de una tarea compleja que no puede abordarse sin contextualizarla en los problemas generales del sistema educativo colombiano, desde las políticas del MEN, hasta el diseño de currículos acordes, por ello mediante el desarrollo de esta investigación se pretende determinar el impacto social del programa de maestría en docencia de la Química (MDQ) en lo relacionado con la profesionalización del diseño curricular en Química (Mora y Parga, 2005) y la necesidad de construir un modelo investigativo sobre la formación permanente del profesorado de química (Parga, Mora y Martínez, 2007).

\section{Abstract}

The permanent formation of teacher's, is at the present time, grateful and even high-priority objective of many educational administrations as well as of numerous institutions and sensitive organisms, official and private, to this necessity. It is a complex task that cannot be approached without contextual in the general problems of the Colombian educational system, from the politicians of the MEN, until the design of in agreement program curriculum, for it by means of the development of this investigation is sought to determine the social impact of the master program in educational of the Chemistry (MDQ) in the related with the professionally of the curriculum design in Chemistry (Mora \& Parga, 2005) and the necessity to build an investigative model on the chemistry faculty's permanent formation (Parga, Mora \& Martinez, 2007).

\section{Palabras claves}

Conocimiento didáctico del contenido curricular, formación permanente del profesorado de química, diseño curricular.

\section{Keywords}

Didactic knowledge of the curricular content, the teacher's of chemistry permanent formation, design curriculum.

\section{Introducción}

Partiendo de que uno de los objetivos fundamentales de la investigación en didáctica de las ciencias hoy, es en torno al diseño curricular y los contenidos de enseñanza, se ha desarrollado una línea que aporta los requerimientos teónicos y metodológicos en este campo, conocida como 
Conocimiento Didáctico del Contenido Curricular CDCC (Mora y Parga, 2007). Esta línea de investigación sobre el CDCC, ha sentado sus bases en hacer una importante diferenciación entre el saber específico de las ciencias y el saber didáctico asociado a su enseñanza, siendo éste un dominio especifico del conocimiento profesional del profesorado, de aquí que el Conocimiento Didáctico del Contenido sea fundamental en la formación inicial y permanente del profesorado de todos los niveles educativos (Parga y Martínez, 2007).

Este proyecto se inscribe dentro de esta línea que se desarrolla en el grupo de investigación ALTERNACIENCLAS, del Departamento de Química de la Universidad Pedagógica Nacional, y dentro de éste, en la línea Didáctica de los Contenidos Curriaulares en Química, en la que se parte del principio que el objeto del desempeño profesional docente es la enseñanza y entomo a ella el diseño y la práctica curricular en las aulas de dase, en el marco de una disaiplina emergente como lo es la didáctica de las ciencias (Parga y Martínez, 2007).

El marco metodológico que orienta esta investigación es constructivista que se orienta en describir e interpretar los fenómenos sociales, y por consiguiente los fenómenos educativos, y se interesa por el estudio de los significados e intenciones de las acciones humanas desde la perspectiva de los propios agentes sociales, ésta se sirve de las palabras, de las acciones y de los documentos orales y escritos para estudiar las situaciones sociales tal como son construidas por los participantes (Torre y Del Rincón, 2003).

\section{Desarrollo}

La formación permanente se impone como una necesidad ineludible en la sociedad actual en todos los ámbitos. Y en la actividad docente esta necesidad se ve aún más acentuada, porque en los profesores recae la responsabilidad de formar a los futuros ciudadanos. Son muchos los programas de formación permanente para profesores que existen en Colombia, impartidos en distintos centros aprobados por el Ministerio de Educación Nacional, uno de los más reconocidos es el de Maestría en Docencia de la Química, ofrecido por la Universidad Pedagógica Nacional desde el año 1987.

Uno de los principales balances de la auto-evaluación del programa en el año 2006 y 2007, al cumplir 21 años, fue sobre la pertinencia de éste en la solución de los problemas de la enseñanza de la química, tanto desde un campo emergente, como lo es la didáctica de las ciencias, como en la conveniencia de los diseños curriculares que se producen en los trabajos de tesis del programa, es decir, si éstos verdaderamente aportan una mejońa en la enseñanza aprendizaje de la química, en el país.

Esto hace que sea oportuno y necesario hacer una investigación, que lleve a recopilar información con los egresados del programa, en auanto al impacto social que éste tiene, que además nos permita vislumbrar sus contribuciones y necesidades en el ámbito educativo, profesional y personal, y asimismo, nos conduzca a caracterizar espećficamente la contribución de la Maestría en Docencia de la Química en el diseño curricular, en cuanto a su trascendencia e implementación en el aula.

Todo esto enmarcado dentro de la necesidad de estudiar los modelos y las concepciones didácticas del profesorado y sus implicaciones en su desarrollo profesional a nivel inicial y permanente, ya que estos aspectos finalmente interfieren en tomo al diseño curricular y determinan las características que tienen los contenidos de enseñanza y cómo se organizan en relación con el conocimiento escolar (Parga y Martínez, 2007). Esto nos permitirá conocer cómo mejorar los espacios académicos propios del programa de MDQ tanto en su estructura curricular, como en las líneas de investigación que ofrece, para corresponder a las necesidades reales de los 
profesores en formación permanente en particular, y de la enseñanza de la química en general, tal como se plantea en los objetivos del programa.

En este sentido el eje fundamental del desarrollo profesional docente se fundamenta en la necesidad de diseñar currículos propios de los espacios académicos, entendiendo el curríaulo como hipótesis progresivas de intervención, innovación e investigación escolar unidas en todo momento con la formación permanente del profesorado (Mora y Parga, 2005).

El Conocimiento Didáctico del Contenido Curricular en Química surge como una línea de investigación que se hace muy importante a la hora de establecer programas de formación inicial y permanente del profesorado de ciencias y se constituye en un referente esencial cuando se evalúa el desempeño profesional del docente en sus diseños aurriaulares (Parga, Mora y Martínez, 2007)

En términos generales, lo que se pretende con esta investigación consiste en determinar el impacto social del programa de MDQ en sus egresados, analizando los diseños que éstos produjeron para la obtención de su tútulo y que está plasmado en el documento final de tesis y además los que producen en su labor docente cotidiana, determinando qué aspectos relacionados con el CDC tuvieron y tienen en cuenta los profesores egresados a la hora de diseñar sus currículos y de esta manera, aportar en la construcción de un programa de formación inicial y permanente de profesores de química más coherente con la profesionalización del diseño curricular.

Los indicadores a analizar en el diseño curricular están planteados a partir del CDC que entendemos a diferencia de otros autores, no solamente como un saber más a adicionar en los profesores en formación inicial y permanente sino como el producto de una combinación de un sistema integrado de los conocimientos, como el conocimiento disciplinar del contenido Cdc (saber académico de referencia, es decir que tanta comprensión tiene de la materia que enseña), conocimiento histórico-epistemológicos, CHE (comprender que y como ha cambiado el conocimiento), conocimiento psicopedagógicos $C p P$ (aprender a pensar en la materia desde la perspectiva del estudiante) y el conocimiento del contexto escolar CCE (aprender a organizar el medio) (Mora y Parga, 2008) todo esto tiene necesariamente implicaciones directas en la formación del profesorado como del tipo de diseños curriculares que formulan.

La línea de investigación sobre el Conocimiento Didáctico del Contenido Curricular en Química, ha centrado su trabajo fundamentalmente en destacar las diferencias entre el saber espećfico de las ciencias y el saber pedagógico y didáctico relacionado con su enseñanza, lo que lo hace necesario en la formación tanto inicial como permanente del profesorado.

Para desarrollar la investigación inicialmente identificaremos el CDC del los profesores egresados del programa de MDQ en lo que denominaremos la primera fase, mediante la caracterización de CDC del egresado en lo plasmado en los diseños curriculares propuestos en las tesis a partir de un análisis documental de los aspectos que tuvieron en cuenta a la hora de diseñar sus propuestas de currículo en sus trabajos de tesis, escogeremos a conveniencia una muestra de los egresados de los últimos cinco años, para realizar la investigación (fase I), en una segunda fase caracterizaremos el CDC de los egresados en su práctica analizando mediante análisis documental, Representaciones de Contenido (ReCo) y Repertorios de Experiencia Profesional Didáctica (ReEpd) (Loughran, Berry, and Mulhall. 2006), como instrumentos complementarios, que conocimientos componentes del CDC tiene en cuenta los Magister cuando seleccionan y diseñan los contenidos de enseñanza en química en su labor docente (fase II), en una última fase determinaremos en el contexto de los egresados el impacto social del programa de MDQ mediante el análisis de factores como la producción científica de los egresados (¿sigue publicando?, cavanza hacia el doctorado?) y el impacto en su entorno (¿sigue haciendo investigación?, ¿pertenece a un grupo de investigación?, ¿participa en eventos?, clidera proyectos?) (fase III) (CNA, 2008). 
En términos generales, lo que se pretende con esta investigación consiste en analizar hasta qué punto el profesor egresado del programa de MDQ impacta su entomo profesional, proyectando y estructurado sus diseños curriculares con el que propuso en su trabajo de investigación para obtener el título de Magister, y de igual manera determinar el impacto social del programa, en la producción científica de sus egresados y en cuanto a la coherencia y pertinencia con su formación permanente a la hora de seleccionar y diseñar los contenidos de enseñanza en química y en su impacto en el entomo en su actividad profesional cotidiana.

Desde estas perspectivas el problema de esta investigación se puede sintetizar en la siguiente pregunta ¿Cuál es el impacto social del programa del programa de maestría en docencia de la Química (MDQ) desde los diseños curriculares propuestos en los documentos de tesis de grado de los egresados del programa?

\section{Conclusiones}

Se pretende cumplir con el objetivo del proyecto y dar solución al problema de investigación, actualmente nos encontramos en la primera fase de la investigación.

\section{Bibliografia}

Concejo Nacional de Acreditación. (2008). Lineamientos para la acreditación de alta calidad de programas de maestría y doctorado. Documento borrador CNA.

Departamento de Química, UPN. (1999). Marco curricular programa de Maestría en Docencia de la Química.

Loughran, J., Berry, A., y Mulhall, P. (2006). Understanding and Developing Saience Teachers' Pedagogical Content Knouledge Rotterdam: Sense Publishers.

Mora, W. y Parga, D. (2008). El Conocimiento Didáctico del Contenido en Química: integración de las Tramas de contenido / histórico - epistemológicas con las Tramas de Contexto / Aprendizaje. En circulación

Mora, W y Parga, D. (2007). Tramas Histórico/Epistemológicas en la evolución de la teoría estructural en química orgánica. Tecnè, Episteme y Didaxis. TED. No. 21. Pp. 100-118.

Mora, W y Parga, D. (2008). El Conocimiento Didáctico del Contenido en Química: De las Tramas Histórico/Epistemológicas a las Tramas de Contexto / Aprendizaje. Tecnè, Episteme $y$ Didaxis. TED. No. 24. Pp. 54-74.

Parga, D. y Martínez, L. (2007). Conocimiento didáctico del contenido curricular en química: una estratega sustentada en el diseño de tramas conceptuales. Proyecto de investigación UPN-CIUPDQU-025-07.

Parga, Mora y Martínez. (2007). El conocimiento didáctico del contenido como programa de investigación: un contexto para la enseñanza de la química. Memorias del III congreso de formación de profesores de ciencias. Revista del la facultad de ciencia y tecnología. Universidad Pedagógica Nacional, Tecne, episteme $y$ Didaxis. TED, numero extra.

Perilla, G. y Parga, D. (2007). La investigación sobre el conocimiento didáctico del contenido curricular, el caso en los profesores de química. Memorias del III congreso de formación de profesores de ciencias. Revista del la facultad de ciencia y tecnología. Universidad Pedagógica Nacional, Tecnè, episteme $y$ Didaxis: TED. Nùmero extra.

Villamizar. D. (2008). Conocimiento Didactico del Contenido Curricular en Química: un estudio con profesores en formacion inicial, UPN, Tesis de pregrado. 